www.nature.com/jhg

\title{
Meiotic behavior of three $D ; G$ Robertsonian translocations: segregation and interchromosomal effect
}

\author{
Ester Anton, Joan Blanco and Francesca Vidal
}

Robertsonian translocations are one of the most frequent reorganizations in humans. Their segregational behavior and their implication in the occurrence of interchromosomal effects (ICEs) has been an important topic of research for the past 10 years. Most of the cases analyzed correspond to rearrangements with chromosomes from the D-group (chromosomes 13, 14 and 15), whereas some rare Robertsonian translocations are scarcely found in the literature, mainly those with both chromosomes from the G-group (chromosomes 21 and 22) and those involving chromosomes from both groups (D;G translocations). Results supporting/ rejecting the existence of the ICE phenomenon have been reported, showing the need for more studies to characterize its distribution. In this study, sperm fluorescent in situ hybridization studies have been performed in three D;G Robertsonian translocation carriers: two men with the translocation $t(14 ; 21)$ and a third individual with the rare $t(13 ; 22)$ reorganization. Segregation and ICE results have been considered in relation to their cytogenetic features and all previously published data. The compiled information discards a particular segregation behavior related to the chromosomes involved. In contrast with this segregational homogeneity, heterogeneous ICE results were observed, indicating a significant but random distribution of such phenomenon.

Journal of Human Genetics (2010) 55, 541-545; doi:10.1038/jhg.2010.67; published online 17 June 2010

Keywords: interchromosomal effect; meiotic segregation; Robertsonian translocations; sperm FISH studies

\section{INTRODUCTION}

Robertsonian translocations were first described in 1916 by WRB Robertson in grasshoppers, ${ }^{1}$ who concluded that the observed $\mathrm{V}$-shaped chromosomes in some individuals corresponded, in fact, to two separate chromosomes in others. Later, these rearrangements were recognized as being one of the most frequent in humans with an incidence of 1.23/1000 in newborns. ${ }^{2}$

These chromosomal reorganizations consist of a fusion of two acrocentric chromosomes at the centromere level to form a derivative chromosome. The human karyotype includes five acrocentric chromosome pairs distributed into two groups: the larger acrocentric chromosomes form group D (that is, chromosomes 13, 14 and 15), whereas the smaller ones constitute group $\mathrm{G}$ (that is, chromosomes 21 and 22). Although Robertsonian translocation can involve any two of these chromosomes, most of them engage chromosomes 13 and $14 .^{2}$

During prophase I, the derivative chromosome and the two respective homologous ones can reach maximum synapsis by forming a trivalent. At anaphase I, this structure may segregate according to three modes: alternate (the two non-translocated chromosomes move to the same pole whereas the derivative segregates to the other), adjacent (the derivative and one of the non-translocated chromosomes segregate to the same pole whereas the remaining non-translocated one goes to the other) and 3:0 (all three chromosomes segregate to the same pole). Only gametes resulting from alternate segregation will be normal or balanced. The other two segregation modes will produce nullisomic or disomic cells for the chromosomes involved.

Although the alternate segregation is classically recognized as the most cytogenetically-favored segregation mode, ${ }^{3}$ some chromosomal factors have been proposed to influence the occurrence of the adjacent segregation: location of breakpoints and chromosomes involved, ${ }^{4,5}$ or a reduced recombination rate in the small chromosome involved. ${ }^{6}$

On the other hand, it has been described that structural reorganization carriers can also produce increased number of unbalanced gametes with numerical abnormalities for chromosomes not related to the rearrangement. These imbalances would be a consequence of disturbances produced by the rearrangements in the correct disjunction of other chromosome pairs. This phenomenon, called interchromosomal effect (ICEs), ${ }^{7}$ is based on the supposed formation of heterologous pairing between the rearranged chromosomes, which often adopt configurations with asynaptic regions, and other chromosomes. ${ }^{8}$

The production of unbalanced gametes by Robertsonian translocation carriers represents a source of fertility problems as they can transmit chromosomal abnormalities to the offspring or lead to miscarriages. The reduction in the reproductive fitness of these males can also entail abnormal seminal parameters, which might be a consequence of alterations in the meiotic process produced by the 
Table 1 Segregation results compiled from previously published data from Robertsonian translocation carriers

\begin{tabular}{|c|c|c|c|c|c|c|}
\hline Rearrangements & Symmetry & Alternate $\bar{x}(\%) \pm s . d$. & Adjacent $\bar{x}(\%) \pm$ s.d. & $3: 0 / 2 n \bar{x}(\%) \pm s . d$. & Other $\bar{x}(\%) \pm s . d$. & No. of cases \\
\hline $\operatorname{der}(13 ; 15)$ & DD & $82.2 \pm 6.8$ & $17.2 \pm 6.5$ & $0.5 \pm 0.4$ & 0.0 & $6^{5,18,19,22}$ \\
\hline $\operatorname{der}(13 ; 21)$ & $D G$ & $87.7 \pm 1.1$ & $11.4 \pm 0.4$ & $0.6 \pm 0.4$ & $0.4 \pm 0.5$ & $2^{21,23}$ \\
\hline $\operatorname{der}(13 ; 22)$ & $\mathrm{DG}$ & 86.7 & 12.8 & 0.5 & 0.0 & $1^{24}$ \\
\hline $\operatorname{der}(14 ; 21)$ & $D G$ & $85.5 \pm 8.3$ & $13.2 \pm 6.8$ & $0.4 \pm 0.5$ & $3.1 \pm 5.1$ & $9^{15,19,25-28}$ \\
\hline $\operatorname{der}(21 ; 22)$ & GG & $82.0 \pm 15.5$ & $16.4 \pm 14.0$ & $0.5 \pm 0.4$ & 4.0 & $4^{18,19,30,31}$ \\
\hline Total & & $84.5 \pm 6.3$ & $14.6 \pm 5.8$ & $0.6 \pm 0.7$ & $1.0 \pm 1.9$ & 67 \\
\hline
\end{tabular}

Abbreviations: max, maximum; min, minimum.

Table 2 ICE results compiled from previously published Robertsonian translocation carriers

\begin{tabular}{lcc}
\hline Rearrangements & Cases with ICE n (\%) & Total cases literature \\
\hline $\operatorname{der}(13 ; 14)$ & $14(60.9)$ & $23^{16,20,21,32-35}$ \\
$\operatorname{der}(13 ; 15)$ & $1(100)$ & $1^{35}$ \\
$\operatorname{der}(13 ; 21)$ & $1(25)$ & $4^{21,23,34,36}$ \\
$\operatorname{der}(13 ; 22)$ & $1(100)$ & $1^{37}$ \\
$\operatorname{der}(14 ; 21)$ & $1(50)$ & $2^{26,33}$ \\
$\operatorname{der}(14 ; 22)$ & $1(50)$ & $2^{21,34}$ \\
Total & $19(57.6)$ & 33 \\
\hline
\end{tabular}

Abbreviation: ICE, interchromosomal effect.

rearrangement. As a result, the incidence of Robertsonian translocation carriers among infertile males is up to eight times higher than that in the general population $(0.78 \%),{ }^{9}$ and thus, they constitute a frequent group of patients who seek genetic reproductive advice in specialized centers.

In the literature, segregation patterns have been analyzed in 67 Robertsonian translocation carriers (Table 1) and although a substantial amount of data has been gathered from these studies, most of them refer to the symmetric 13;14 translocations. ${ }^{17-19,21}$ Segregation studies in other Robertsonian translocations are much more limited, especially those obtained from rearrangements involving chromosomes from groups D and G (less than 25\%). Moreover, in this last group, some Robertsonian translocations are represented in a sole report, as are the cases of translocations $13 ; 22^{24}$ and $15 ; 22 .{ }^{29}$

Regarding the occurrence of ICEs, aneuploidy analyses have been performed in 33 Robertsonian translocation carriers. Among them, 24 were D;D rearrangements and $96 \%$ corresponded to $13 ; 14$ carriers (Table 2).

In this study segregation, ICE studies for chromosomes $18, \mathrm{X}$ and $\mathrm{Y}$ have been performed in two men with karyotype 45,XY,der(14;21) (q10;q10) and in a carrier of $45, \mathrm{XY}, \operatorname{der}(13 ; 22)(\mathrm{q} 10 ; \mathrm{q} 10)$. Data obtained have been examined and considered in relation to the previously published studies in the literature.

\section{MATERIALS AND METHODS}

Three unrelated male carriers of Robertsonian translocations who showed oligoasthenozoospermia ${ }^{38}$ were recruited when they consulted for infertility (Table 3): P1 was 28 years old and showed a 45,XY,der(13;22)(q10;q10) karyotype, P2, aged 41 years, and P3, aged 43 years, had a 45,XY,der(14;21) (q10;q10) karyotype, as well as $\mathrm{P} 3$ who was aged 43 years. All of them gave their written informed consent to participate in the study, which was approved by our Institutional Ethics Committee.

\section{Semen sample processing}

Semen samples were fixed in methanol:acetic acid (3:1) and were spread on a slide. Sperm nuclei were decondensed by slide incubation in $5 \mathrm{~mm}$ dithiothreitol, as described by Vidal et al. ${ }^{39}$ and as detailed by Sarrate et al. ${ }^{40}$ Afterwards, preparations were processed for fluorescent in situ hybridization (FISH) analysis.

\section{FISH}

A dual-color FISH was used to assess the segregation behavior of each rearrangement. For P1, a combination of a subtelomeric probe specific for the 13q region (TelVysion 13q Spectrum Orange; Vysis; Downers Grove, IL, USA) and a locus-specific probe for the 22q11.2 region (LSI 22, bcr, Spectrum Green; Vysis) were used to identify the segregation products. For P2 and P3, a combination of a locus-specific probe for chromosome 21 (LSI 21, 21q22.13q22.2, Spectrum Orange; Vysis) and a subtelomeric probe specific for the $14 \mathrm{q}$ region (TelVysion $14 \mathrm{q}$ Spectrum Green; Vysis) were used.

Regarding ICE evaluation, diploidy and disomy frequencies for chromosomes 18, $\mathrm{X}$ and $\mathrm{Y}$ were analyzed using a multicolor probe panel for chromosomes 18 (CEP18, D18Z1, Spectrum Aqua), X (CEPX, DXZ1, Spectrum Green) and Y (CEPY, DYZ3, Spectrum Orange) from the Vysis AneuVysion Multicolor DNA Probe Kit (Vysis).

The protocol for probes and sample denaturation, incubation and detection was standardized in our laboratory according to the manufacturer's instructions (Vysis).

\section{Statistical analysis}

Data obtained were statistically analyzed using SPSS 13.0 (SPSS; Chicago, IL, USA) as per the guidelines ofthe Statistical Service of the Universitat Autònoma de Barcelona.

ICE results were compared with control data previously published from our group. $^{40}$

Differences were considered to be statistically significant when $P<0.05$.

\section{RESULTS}

The results obtained in the segregation analysis are described in Table 4. All three carriers showed a very similar segregation pattern, with a preferential alternate segregation mode (range 78.5-85.5\%) followed by the adjacent segregation mode (range 13.9-20.9\%). The percentage of gametes with complementary adjacent contents (nullisomies versus disomies for chromosomes implicated in the reorganization) were not significantly different in any carrier except for P1, who presented a higher production of gametes with chromosome 22 disomies than with chromosome 22 nullisomies $(P=0.007$; Table 4). Taking the three individuals as a group, these differences were 
Table 3 Clinical characteristics of patients' sperm parameters

\begin{tabular}{llllrr}
\hline Patients & Karyotype & Age & Seminogram & Density $\left(10^{6} \mathrm{~m}^{-1}\right)$ & \% Total motility \\
\hline P1 & $45, \mathrm{XY}, \operatorname{der}(13 ; 22)(\mathrm{q} 10 ; \mathrm{q} 10)$ & 28 & Oligoasthenozoospermia & 0.9 \\
P2 & $45, \mathrm{XY}, \operatorname{der}(14 ; 21)(\mathrm{q} 10 ; \mathrm{q10})$ & 41 & Oligoasthenozoospermia & 1 & 5 \\
P3 & $45, \mathrm{XY}, \operatorname{der}(14 ; 21)(\mathrm{q} 10 ; \mathrm{q10})$ & 43 & Oligoasthenozoospermia & 4.6 & 1 \\
\hline
\end{tabular}

Table 4 Segregation analysis results in the three Robertsonian translocation carriers

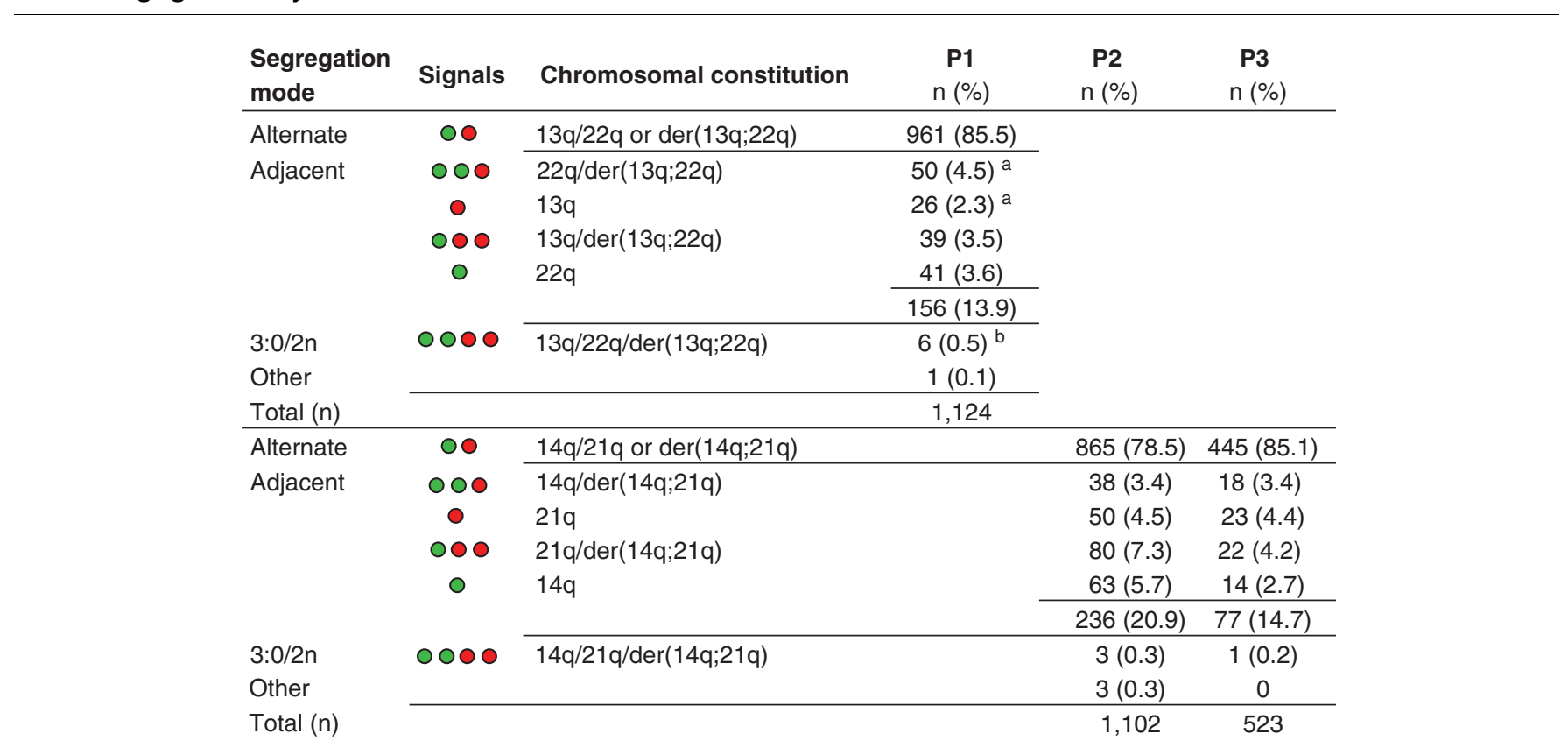

avalues with the same superscript differ significantly $(P<0.05)$

bSignificant differences versus control population $(P<0.05) .^{40}$

Table 5 Results of ICE studies for chromosomes $18, X$ and $Y$

\begin{tabular}{lcccrrr}
\hline Patients & Haploid $\mathrm{n}(\%)$ & Sex chromosome disomy $\mathrm{n}(\%)$ & Disomy $18 \mathrm{n}(\%)$ & Diploidy $\mathrm{n}(\%)$ & Other $\mathrm{n}(\%)$ & Total \\
\hline P1 & $10362(98.87)$ & $40(0.38)^{\mathrm{a}}$ & $3(0.03)$ & $36(0.34)^{\mathrm{a}}$ & $39(0.37)$ & 10480 \\
P2 & $10173(99.57)$ & $17(0.17)$ & $9(0.09)$ & $9(0.09)$ & $9(0.09)$ & 10217 \\
P3 & $2339(99.74)$ & $1(0.04)$ & $2(0.09)$ & $2(0.09)$ & 0 & 2347 \\
Control data $^{40}$ & $63150(98.96)$ & $120(0.19 \%)$ & $22(0.03 \%)$ & $123(0.19 \%)$ & $3(0.01 \%)$ & 63811 \\
\hline
\end{tabular}

Abbreviation: ICE, interchromosomal effect.

asignificant differences versus control data $(P<0.05) .{ }^{40}$

not significantly different either for chromosome $22(P=0.669)$ or for chromosome $13(P=0.161)$.

The FISH methodology did not allow us to differentiate between 3:0 segregations and diploid spermatozoa, as both chromosomal constitutions were coincident in showing two hybridization signals for the two probes used. These products accounted for $0.53 \%$ of sperm analyzed in carrier $\mathrm{P} 1$ and $0.27 \%$ in carrier P2, whereas no spermatozoa with this chromosomal content were identified in carrier P3 (Table 4).

In the ICE study, only P1 presented significant increases in gametes with numerical abnormalities. These increases were with regard to sex chromosome aneuploidies $(0.38 \% ; P<0.001)$ and diploidies $(0.34 \%$; $P=0.003$ ) (Table 5).

Diploidy rates from ICE studies were compared with spermatozoa scored as diploid/3:0 in the segregation analysis. We did not find significant differences $(P>0.05)$.

\section{DISCUSSION}

\section{Segregation behavior}

The segregation patterns observed in all three Robertsonian translocation carriers were very similar, with the main production of normal/ balanced spermatozoa resulting from an alternate segregation. These results agree with the classical cytogenetic models which proposed the alternate segregation mode as the most favored chromosome distribution in a trivalent segregation. ${ }^{3}$

Among unbalanced gametes, most of them corresponded to adjacent segregation as only less than $0.5 \%$ were classified as 3:0/2n. This behavior agrees with the 67 previously published segregation studies performed in Robertsonian translocation carriers, which report a clear predominance of alternate segregation as opposed to adjacent segregation.

In particular, the previous nine segregation reports performed in der(14;21) carriers describe an average ( \pm s.d.) frequency of alternate 
products of $85.5 \pm 8.3$ and adjacent products of $13.2 \pm 6.8$. Patterns obtained in P2 and P3 would fit within this population. In fact, the statistical comparison of our results with the published data does not show significant differences, neither for the alternate segregation (Mann-Whitney test; $P=0.3273$ ) nor for the adjacent one (Mann-Whitney test; $P=0.2182$ ).

With regard to $\mathrm{P} 1$, our results are very similar to those reported in the sole der $(13 ; 22)$ carrier analyzed in the literature. ${ }^{24}$ No statistical differences exist between these two cases $\left(\chi^{2}\right.$-test; $\left.P=0.589\right)$.

Some authors have related a major production of gametes with an adjacent content to Robertsonian translocations with different breakpoints and chromosomes involved. ${ }^{4-6}$ Nevertheless, when we compare the entire published data, we cannot observe significant differences regarding the frequencies of alternate or adjacent segregation among Robertsonian translocation carriers depending on the combination of acrocentric chromosomes involved (that is, DD, DG and GG rearrangements; test de Kruskal-Wallis; $P=0.3230$ and $P=0.9089$, respectively).

According to the compiled results presented in Table 1, the segregation variation among the different kinds of Robertsonian translocations is higher within the same group than among the different assortment analyzed. However, this variation is small and could be associated with other non-chromosomal factors, such as the particular characteristics of each individual, technical aspects of the methodologies used and the number of spermatozoa evaluated in each study. The sample size of the studies performed describes a wide range which goes from 24 to 16578 sperm analyzed per case.

The statistical power of the studies with a small number of scored sperm is lower and results in a major distortional effect. In particular, the two cases that are the most deviated from the population average are those corresponding to the two sperm FISH studies with a small sample size..$^{30,31}$

\section{ICE}

The incorporation of ICE studies requires a previous selection of the chromosomes to be analyzed as indicators of the occurrence of such a phenomenon. In the literature, data for autosomes $1,6,13,15,16$, $17,18,21,22$ and sex chromosomes are reported. Nevertheless, almost all cases coincide in combining the analysis of at least one autosome with sex chromosomes. To be able to integrate our data among these results, our ICE study was designed to concur with these conditions (analysis of chromosomes 18, X and Y).

None of our analyzed $\operatorname{der}(14 ; 21)$ carriers showed significant increases in diploidies or numerical abnormalities for the chromosomes analyzed. However, P3 showed increased percentage of sex chromosome disomies and diploidies versus the control population.

In the literature (Table 2), one of the two ICE studies performed in $\operatorname{der}(14 ; 21)$ carriers reported significant aneuploidy increases for chromosome 1 and diploidies, ${ }^{26}$ whereas the single $\operatorname{der}(13 ; 22)$ analyzed $^{37}$ did not show significant increases for the chromosomes analyzed (chromosome 21 and diploidies).

Further ICE studies have been performed in other Robertsonian translocation carriers although most of them correspond to der $(13 ; 14)$ carriers (Table 2). Nonetheless, cases with a significant ICEs can be detected in all groups of Robertsonian translocations analyzed, representing $58 \%$ of the total (Table 2). The incidences described among the compiled data are higher than those of not only the general population but also the population of infertile males with a normal karyotype (around 14\%). ${ }^{40}$ This fact contributes to corroborate the controversial relation between the presence of such numerical anoma- lies in reorganization carriers and a disturbance effect produced by the rearrangement, as has been commented elsewhere. ${ }^{41}$

The frequency of cases with significant ICEs does not show a preferential distribution of this effect according to the groups of Robertsonian translocation analyzed (Kruskal-Wallis test; $P=0.1436$ ) or its symmetry (Fisher's exact test; $P=0.5320$ ). In reciprocal translocation carriers, a similar circumstance has been noticed as the distribution of cases with a significant ICEs among this population could not have been associated with any specific cytogenetic feature. ${ }^{42}$

Therefore, the occurrence of this phenomenon represents a factual genetic reproductive risk for Robertsonian translocation carriers, although the risk-classification criterion has not been established for the time being.

\section{ACKNOWLEDGEMENTS}

This work was funded by project 2009 SGR-282 (Agència de Gestió d'Ajuts Universitaris i de Recerca de la Generalitat de Catalunya, SPAIN) and project 180034 (Universitat Autònoma de Barcelona, Spain).

1 Robertson, W. R. B. Chromosome studies I. Taxonomic relationships shown in the chromosomes of Tettigidae and Acrididae: V-shaped chromosomes and their significance in Acrididae, Locustidae, and Grykllidae: chromosomes and variation. J. Morphol. 27, 179-331 (1916).

2 Nielsen, J. \& Wohlert, M. Chromosome abnormalities found among 34,910 newborn children: results from a 13-year incidence study in Arhus, Denmark. Hum. Genet. 87, 81-83 (1991).

3 Sybenga, J. General Cytogenetics (North-Holland Publishing Company, Amsterdam, The Netherlands, 1975).

4 Moradkhani, K., Puechberty, J., Bhatt, S., Vago, P., Janny, L., Lefort, G. et al. Meiotic segregation of rare Robertsonian translocations: sperm analysis of three $t(14 q ; 22 q)$ cases. Hum. Reprod. 21, 1166-1171 (2006).

5 Moradkhani, K., Puechberty, J., Bhatt, S., Lespinasse, J., Vago, P., Lefort, G. et al. Rare Robertsonian translocations and meiotic behaviour: sperm FISH analysis of $t(13 ; 15)$ and $\mathrm{t}(14 ; 15)$ translocations: a case report. Hum. Reprod. 21, 3193-3198 (2006).

6 Munné, S., Escudero, T., Sandalinas, M., Sable, D. \& Cohen, J. Gamete segregation in female carriers of Robertsonian translocations. Cytogenet. Cell Genet. 90, 303-308 (2000).

7 Lejeune, J. Autosomal disorders. Pediatrics 32, 326-337 (1963).

8 Guichaoua, M. R., Quack, B., Speed, R. M., Noel, B., Chandley, A. C. \& Luciani, J. M. Infertility in human males with autosomal translocations: meiotic study of a $14 ; 22$ Robertsonian translocation. Hum. Genet. 86, 162-166 (1990).

9 De Braekeleer, M. \& Dao, T. N. Cytogenetic studies in male infertility: a review. Hum. Reprod. 6, 245-250 (1991).

10 Pellestor, F., Sele, B. \& Jalbert, H. Chromosome analysis of spermatozoa from a male heterozygous for a 13;14 Robertsonian translocation. Hum. Genet. 76, 116-120 (1987).

11 Martin, R. H. Cytogenetic analysis of sperm from a male heterozygous for a $13 ; 14$ Robertsonian translocation. Hum. Genet. 80, 357-361 (1988).

12 Ogawa, S., Araki, S., Araki, Y., Ohno, M. \& Sato, I. Chromosome analysis of human spermatozoa from an oligoasthenozoospermic carrier for a 13;14 Robertsonian translocation by their injection into mouse oocytes. Hum. Reprod. 15, 1136-1139 (2000).

13 Escudero, T., Lee, M., Carrel, D., Blanco, J. \& Munne, S. Analysis of chromosome abnormalities in sperm and embryos from two $45, \mathrm{XY}, \mathrm{t}(13 ; 14)(\mathrm{q} 10 ; \mathrm{q} 10)$ carriers. Prenat. Diagn. 20, 599-602 (2000).

14 Morel, F., Roux, C. \& Bresson, J. L. FISH analysis of the chromosomal status of spermatozoa from three men with $45, \mathrm{XY}$, $\operatorname{der}(13 ; 14)(\mathrm{q} 10 ; \mathrm{q} 10)$ karyotype. Mol. Hum. Reprod. 7, 483-488 (2001).

15 Frydman, N., Romana, S., Le Lorc'h, M., Vekemans, M., Frydman, R. \& Tachdjian, G. Assisting reproduction of infertile men carrying a Robertsonian translocation. Hum. Reprod. 16, 2274-2277 (2001).

16 Anton, E., Blanco, J., Egozcue, J. \& Vidal, F. Sperm FISH studies in seven male carriers of Robertsonian translocation $\mathrm{t}(13 ; 14)(\mathrm{q} 10 ; \mathrm{q} 10)$. Hum. Reprod. 19, 1345-1351 (2004).

17 Roux, C., Tripogney, C., Morel, F., Joanne, C., Fellmann, F., Clavequin, M. C. et al. Segregation of chromosomes in sperm of Robertsonian translocation carriers. Cytogenet. Genome Res. 111, 291-296 (2005).

18 Brugnon, F., Van Assche, E., Verheyen, G., Sion, B., Boucher, D., Pouly, J. L. et al. Study of two markers of apoptosis and meiotic segregation in ejaculated sperm of chromosomal translocation carrier patients. Hum. Reprod. 21, 685-693 (2006).

19 Ogur, G., Van Assche, E., Vegetti, W., Verheyen, G., Tournaye, H., Bonduelle, M. et al. Chromosomal segregation in spermatozoa of 14 Robertsonian translocation carriers. Mol. Hum. Reprod. 12, 209-215 (2006). 
20 Kekesi, A., Erdei, E., Torok, M., Dravucz, S. \& Toth, A. Segregation of chromosomes in spermatozoa of four Hungarian translocation carriers. Fertil. Steril. 88, 212 e215-211 (2007).

21 Chen, Y., Huang, J., Liu, P. \& Qiao, J. Analysis of meiotic segregation patterns and interchromosomal effects in sperm from six males with Robertsonian translocations. J. Assist Reprod. Genet. 24, 406-411 (2007).

22 Pellestor, F. Analysis of meiotic segregation in a man heterozygous for a $13 ; 15$ Robertsonian translocation and a review of the literature. Hum. Genet. 85, 49-54 (1990).

23 Hatakeyama, C., Gao, H., Harmer, K. \& Ma, S. Meiotic segregation patterns and ICSI pregnancy outcome of a rare $(13 ; 21)$ Robertsonian translocation carrier: a case report. Hum. Reprod. 21, 976-979 (2006).

24 Anahory, T., Hamamah, S., Andreo, B., Hedon, B., Claustres, M., Sarda, P. et al. Sperm segregation analysis of a $(13 ; 22)$ Robertsonian translocation carrier by FISH: a comparison of locus-specific probe and whole chromosome painting. Hum. Reprod. 20, 1850-1854 (2005).

25 Balkan, W. \& Martin, R. H. Segregation of chromosomes into the spermatozoa of a man heterozygous for a 14;21 Robertsonian translocation. Am. J. Med. Genet. 16, 169-172 (1983).

26 Rousseaux, S., Chevret, E., Monteil, M., Cozzi, J., Pelletier, R., Delafontaine, D. et al Sperm nuclei analysis of a Robertsonian $\mathrm{t}(14 \mathrm{q} 21 \mathrm{q})$ carrier, by FISH, using three plasmids and two YAC probes. Hum. Genet. 96, 655-660 (1995).

27 Tang, Y. P., Liu, X. S., Liu, Y., Yang, Z. R., Chen, Y. \& Xiong, C. L. Somatic cell and sperm cell cytogenetics in a patient with $\mathrm{t}(14 ; 21)$. Yi Chuan Xue Bao. 33, 488-494 (2006).

28 Honda, H., Miharu, N., Samura, O., He, H. \& Ohama, K. Meiotic segregation analysis of a 14;21 Robertsonian translocation carrier by fluorescence in situ hybridization. Hum. Genet. 106, 188-193 (2000).

29 Martin, R. H., Ko, E. \& Hildebrand, K. Analysis of sperm chromosome complements from a man heterozygous for a Robertsonian translocation 45,XY,t(15q;22q). Am. J. Med. Genet. 43, 855-857 (1992).

30 Syme, R. M. \& Martin, R. H. Meiotic segregation of a 21;22 Robertsonian translocation. Hum. Reprod. 7, 825-829 (1992).

31 Mennicke, K., Diercks, P., Schlieker, H., Bals-Pratsch, M., al Hasani, S., Diedrich, K. et al. Molecular cytogenetic diagnostics in sperm. Int. J. Androl. 20 (Suppl 3), 11-19 (1997).
32 Morel, F., Fellmann, F., Roux, C. \& Bresson, J. L. Meiotic segregation analysis by FISH investigation of spermatozoa of a $46, \mathrm{Y}, \operatorname{der}(\mathrm{X}), \mathrm{t}(\mathrm{X} ; \mathrm{Y})$ (qter->p22::q11-> qter) carrier. Cytogenet. Cell Genet. 92, 63-68 (2001).

33 Douet-Guilbert, N., Bris, M. J., Amice, V., Marchetti, C., Delobel, B., Amice, J. et al. Interchromosomal effect in sperm of males with translocations: report of 6 cases and review of the literature. Int. J. Androl. 28, 372-379 (2005).

34 Baccetti, B., Collodel, G., Marzella, R., Moretti, E., Piomboni, P., Scapigliati, G. et al. Ultrastructural studies of spermatozoa from infertile males with Robertsonian translocations and 18, X, Y aneuploidies. Hum. Reprod. 20, 2295-2300 (2005).

35 Machev, N., Gosset, P., Warter, S., Treger, M., Schillinger, M. \& Viville, S. Fluorescence in situ hybridization sperm analysis of six translocation carriers provides evidence of an interchromosomal effect. Fertil. Steril. 84, 365-373 (2005).

36 Kirkpatrick, G., Ferguson, K. A., Gao, H., Tang, S., Chow, V., Yuen, B. H. et al. A comparison of sperm aneuploidy rates between infertile men with normal and abnormal karyotypes. Hum. Reprod. 23, 1679-1683 (2008).

37 Blanco, J., Egozcue, J. \& Vidal, F. Interchromosomal effects for chromosome 21 in carriers of structural chromosome reorganizations determined by fluorescence in situ hybridization on sperm nuclei. Hum. Genet. 106, 500-505 (2000).

38 World Health Organization. WHO Laboratory Mannual for the Examination of Human Semen and Semen-Cervical Mucus Interaction, 4th edn. (Cambridge University Press, Cambridge, UK, 1999).

39 Vidal, F., Moragas, M., Catala, V., Torello, M. J., Santalo, J., Calderon, G. et al. Sephadex filtration and human serum albumin gradients do not select spermatozoa by sex chromosome: a fluorescent in-situ hybridization study. Hum. Reprod. 8, 1740-1743 (1993).

40 Sarrate, Z., Vidal, F. \& Blanco, J. Role of sperm fluorescent in situ hybridization studies in infertile patients: indications, study approach and clinical relevance. Fertil. Steril. 93, 1892-1902 (2010).

41 Anton, E., Vidal, F. \& Blanco, J. Role of sperm FISH studies in the genetic reproductive advice of structural reorganization carriers. Hum. Reprod. 22, 2088-2092 (2007).

42 Anton, E., Vidal, F. \& Blanco, J. Reciprocal translocations: tracing their meiotic behavior. Genet. Med. 10, 730-738 (2008). 